\title{
Near-source characteristics of two-phase gas-solid outbursts in roadways
}

\author{
Aitao Zhou ${ }^{1,2,3} \cdot$ Meng Zhang ${ }^{2} \cdot$ Kai Wang ${ }^{1,2} \cdot$ Derek Elsworth $^{3}$
}

Received: 26 April 2020/Revised: 3 June 2020/Accepted: 3 September 2020/Published online: 29 September 2020

(C) The Author(s) 2020

\begin{abstract}
Coal and gas outbursts compromise two-phase gas-solid mixtures as they propagate as shock waves and flows from their sources. Propagation is influenced by the form of the outburst, proximity to source, the structure and form of the transmitting roadways and the influence of obstacles. The following characterizes the propagation of coal and gas outbursts as two-phase gas-solid flows proximal to source where the coupled effects of pulverized coal and gas flows dominate behavior. The characteristics of shock wave propagation and attenuation were systematically examined for varied roadway geometries using experiments and numerical models. The results demonstrate that the geometry of roadway obstructions is significant and may result in partial compression and sometimes secondary overpressurization in blocked and small corner roadways leading to significant attenuation of outburst shock waves. The shock waves attenuate slowly in both straight and abruptly expanding roadways and more significantly in T-shaped roadways. The most significant attenuation appears in small angle corners and bifurcations in roadways with the largest attenuation occurring in blocked roadways. These results provide basic parameters for simplifying transport in complex roadway networks in the far-field, and guidance for the design of coal and gas outburst prevention facilities and emergency rescue.
\end{abstract}

Keywords Coal and gas outburst · Proximity to source $\cdot$ Shock wave propagation $\cdot$ Two-phase gas-solid flow $\cdot$ Outburst prevention

\section{Introduction}

Coal and gas outbursts in underground coal mines are capable of rapidly and energetically ejecting significant masses of coal and gas into the roadways and stopes ( $\mathrm{Yu}$ 1992; Zhao et al. 2020). These bumps and bursts scavenge

Kai Wang

safety226@126.com

1 Beijing Key Laboratory for Precise Mining of Intergrown Energy and Resources, China University of Mining and Technology (Beijing), Beijing 100083, China

2 School of Emergency Management and Safety Engineering, China University of Mining and Technology (Beijing), Beijing 100083, China

3 Department of Energy and Mineral Engineering, EMS Energy Institute and G3 Center, Pennsylvania State University, University Park, PA, USA their kinetic energy from elastic strain and gas expansion energy. The resulting events compress the air in roadways in the form of outburst shock waves and gas flows, may result in serious damage to the underground ventilation system and cause casualties and may also contribute to the occurrence of secondary accidents such as gas explosions (Yang and Zhang 2012). Therefore, it is of great significance to understand the modes of occurrence and attenuation of the outburst at source in order to effectively prevent and control coal and gas outbursts. There are many factors influencing shock waves and the propagation of gas flows (Zhao et al. 2018). These include roadway structure, obstacles, coal wall roughness, airflow components and temperature, among others. Of these, roadway structure and obstacles play the most significant role. Few studies describe the propagation characteristics of outburst shock wave at source, as a precursor to define its distal effect. An outburst at the Zhongliangshan Coal Mine, Sichuan, China 
in 1977 recorded a static pressure head of gas and pulverized coal flow in the initial stage of the outburst at 0.3 - 0.6 MPa (Wang and Yu 2005)—confirming that the outburst shock waves entrain significant destructive energy. Attenuation mechanisms of shock waves have been deduced (Cheng and Chen 2000; Cheng et al. 2004) including the use of physical and numerical models (Otuonye and Sheng 1994). These have defined the principal characteristics of shock wave propagation in roadways (Miao et al. 2013) including the influence of the gas phase (Wang et al. 2012, 2011; Zhou and Wang 2017). The outburst shock wave is known to result from the mobilization of a large amount of pulverized coal carried by gas (Wu et al. 2019; Xue et al. 2015). However, none of the prior studies considered the interaction between the pulverized coal and gas flow. In addition, prior studies have neglected any in-depth analysis of the influence of the roadway structure on outburst shock waves. In the following we analyzed the coupling between pulverized coal and gas flows with full consideration of the influence of pulverization on the development of outbursts. We applied appropriate initial and boundary conditions to this twophase gas-solid flow model of pulverized coal and gas to explore propagation characteristics of the outburst shock waves and their interaction with different roadway structures and obstacles to define the spectrum of destructive impacts. On the one hand, the laws of near-source twophase gas-solid flow can guide the design of outburst prevention facilities such as outburst prevention damper. When the outburst occurs, the shock wave causes strong damage to the anti-outburst damper and anti-counterflow device, and leads to the disturbance of mine ventilation system. The study of outburst dynamic attenuation characteristics can reveal the level of shock overpressure at structures such as outburst dampers, and provide theoretical guidance for the strength and installation position design of outburst dampers. On the other hand, it can guide the emergency rescue after the accident. One is to use the attenuation characteristics of the outburst shock wave to judge whether the existing anti outburst damper fails after the outburst accident. The second is to provide initial parameters for gas migration in far-field and predict the scope of disaster threat, so as to guide emergency rescue after disaster.

\section{Near-source two-phase gas-solid flow model}

Although the mechanism of coal and gas outbursts is still unclear, many outburst case studies confirm intense coal fragmentation and pulverization resulting from rapid gas desorption and expansion together with rapid ejection of the pulverized coal and gas as a two-phase flow (Guo et al.
2016; Jin et al. 2018; Sun et al. 2018; Xu and Jiang 2017). At macroscale this solid-fluid interaction may be regarded as an equivalent fluid mixture responding with a rheology dependent on its solid content. This is convenient as this equivalent fluid may be readily accommodated in sophisticated models for computational fluid dynamics (Berger et al. 2009; Chaudhuri et al. 2012; Xue et al. 2011). The intense ejection of the outburst lessens as it travels progressively further from the source (Biamino et al. 2013). Distal from its source, the two-phase flow can be regarded as homogeneous fluid mixed with solid particles. Similarly, at the time of the outburst, the action of the high-pressure gas and stress is presumed to result in a well-mixed gassolid mixture (Igra and Igra 2016; Lin et al. 2016; Uystepruyst and Monnoyer 2015). Figure 1 shows a simplified two-dimensional model of an outburst in a roadway comprising an outburst chamber and its exit to the roadway. The chamber is of length $L$ and height $H$, and the roadway extends along the $x$ direction. The roof and floor are set as simple smooth rigid walls and the roadway outlets at atmospheric pressure.

Two interactive forces dominate in two-phase flows. The first is that due to friction and collision between solids, including friction and collision between pulverized coal particles and between particles and the roadway wall. The second is the coupling between particles and gas. The former leads to a resistance effect as the kinetic energy of solid particles is transformed to fracture the particles with the concomitant generation of heat. We simplify this by accommodating only the elastic collision between particles where the collision force between particles $\mathrm{a}$ and $\mathrm{b}$ moving together (ANSYS 2017) is expressed as:

$\vec{p}=-\frac{m_{\mathrm{a}} m_{\mathrm{b}}}{m_{\mathrm{a}}+m_{\mathrm{b}}} \cdot \frac{\mathrm{d}\left(\overrightarrow{u_{\mathrm{b}}}-\overrightarrow{u_{\mathrm{a}}}\right)}{\mathrm{d} t}$

where $m_{\mathrm{a}}$ is the mass of particle a, $m_{\mathrm{b}}$ is the mass of particle $\mathrm{b}, \frac{m_{\mathrm{a}} m_{\mathrm{b}}}{m_{\mathrm{a}}+m_{\mathrm{b}}}$ is a "reduced mass" of particles a and $\mathrm{b}$, and $\overrightarrow{u_{\mathrm{b}}}-\overrightarrow{u_{\mathrm{a}}}$ is the relative velocity between them.

The coal particles have a tendency to settle due to gravity, but the drag force added by the high horizontal velocity of the gas is dominant and resists this with inertia. Thus, the pulverized coal exerts a certain "blocking effect" on the gas flow, in turn, leading to energy consumption in

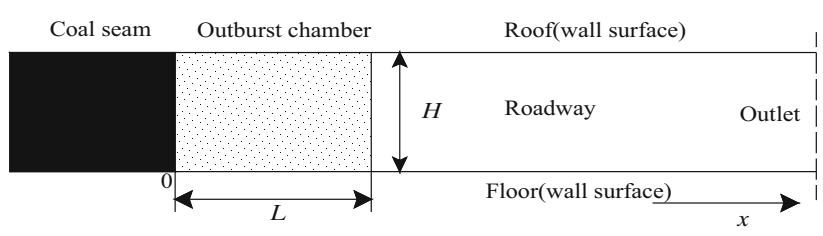

Fig. 1 Schematic model of outburst roadway 


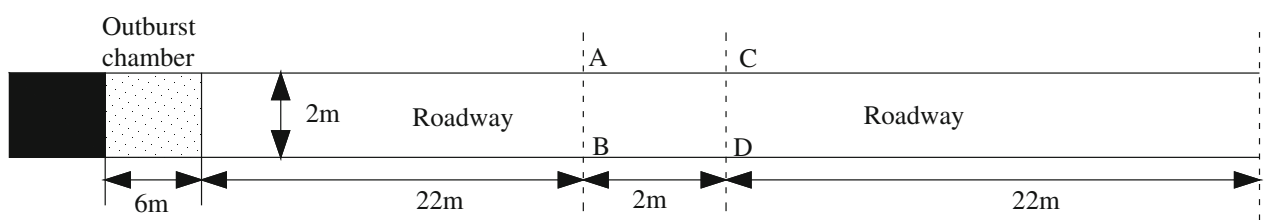

Fig. 2 Geometry of straight roadway

this process. This slip velocity between the two phases is accommodated where:

$\vec{v}_{\mathrm{pq}}=\frac{\tau_{\mathrm{p}}}{f_{\text {drag }}} \frac{\left(\rho_{\mathrm{p}}-\rho_{\mathrm{m}}\right)}{\rho_{\mathrm{p}}} \vec{a}$

where, $\vec{v}_{\mathrm{pq}}$ represents the relative velocity between pulverized coal and gas flow; $\rho_{\mathrm{p}}$ and $\rho_{\mathrm{m}}$ represent the density of pulverized coal and the mixing density respectively; $\vec{a}$ represents the accelerated velocity of the pulverized coal, and $\tau_{\mathrm{p}}$ is the relaxation time of pulverized coal particles, expressed as follows:

$\tau_{\mathrm{p}}=\frac{\rho_{\mathrm{p}} d_{\mathrm{p}}^{2}}{18 \mu_{\mathrm{q}}}$

where, $d_{\mathrm{p}}$ represents the particle diameter of the pulverized coal; $\mu_{\mathrm{q}}$ represents the gas viscosity, and the drag force function $f_{\text {drag }}$ is expressed as:

$f_{\text {drag }}= \begin{cases}1+0.15 R e^{0.687} & R e \leq 1000 \\ 0.0183 R e & R e>1000\end{cases}$

where $R e$ represents the Reynolds number. The specific controlling equations of the model are based on mass conservation, momentum conservation, energy conservation and component conservation. The $k-\varepsilon$ turbulence model is adopted.

\section{Propagation characteristics of energetic two- phase gas-solid flows}

FLUENT was utilized to simulate the two-phase gas-solid flow characteristics in the near-field of roadways close to the outburst source. The model comprises two parts: an outburst chamber containing a high-pressure two-phase mixture and roadways in which the outburst shock wave propagates. As noted previously, the outburst area is replaced by a high-pressure chamber, of length $L$. A fixed volume of gas and a pre-defined particle size distribution of pulverized coal is set into this chamber. The volume fraction of pulverized coal is $V$, and it is presumed fully mixed with the gas and evenly distributed in the outburst chamber. Since the objective of the study is to determine the various impacts of roadway structure on the transmission of the shock wave, the particle diameter is fixed to a default value of $0.01 \mathrm{~mm}$ in the simulations. The initially static gas is acted upon by a high-pressure source at original outburst pressure $P_{1}$ with the temperature of gas and air assumed to be $300 \mathrm{~K}$. Compared to the outburst airflow, the actual normal gas concentration and air velocity within the roadways are small and therefore neglected. Therefore, the initial air velocity in the outburst roadway is set as $0 \mathrm{~m} /$ $\mathrm{s}$, the gas concentration set as null, and the initial pressure as atmospheric pressure. In addition, the volume fraction of reflux at the pressure outlet is null, eliminating reflux in the evolving high-speed airflow. The specific turbulence mode is realized by defining the hydraulic radius and intensity. It should be noted that the shock wave front is not aligned parallel with the cross section, i.e., the pressure across the cross section is not uniform, especially in the blocked roadway. Thus, the pressure on the monitoring surface is taken as the average pressure on the cross section.

\subsection{Propagation characteristics in straight roadways}

Figure 2 shows the outburst geometry linked to a straight roadway. The outburst chamber is $6 \mathrm{~m}$ long, $2 \mathrm{~m}$ high and is attached to a roadway of length $46 \mathrm{~m}$. The conditions are set with an initial pressure in the outburst chamber of $1 \mathrm{MPa}$ and the volume fraction of the pulverized coal at $5 \%$ (all following simulations follow the same conditions). The characteristics of shock wave propagation are observed at two sections at $22 \mathrm{~m}$ (section $A B$ ) and $24 \mathrm{~m}$ (section $C D$ ) downstream from the outburst-recording the variation of the shock pressure and other parameters with time. The simulation results are shown in Fig. 3.

Apparent from Fig. 3 is that the distribution of flow pressure and gas (Fig. 3a, b) is initially concentrated in the outburst chamber at $0.02 \mathrm{~s}$. This is due to the incomplete pressure relief and the incomplete expansion of gas in the outburst area. Only a small part of the pressure is released within the first $\sim 10 \mathrm{~m}$. The velocity of airflow here reaches $190 \mathrm{~m} / \mathrm{s}$, while the gas in the chamber does not accelerate due to the negative pressure (Fig. 3c). Simultaneously, the pressure gradient is a maximum and the gas and pulverized coal undergo a large acceleration. By $0.14 \mathrm{~s}$, the front of the shock wave has reached the exit of roadway and the velocity of the gas-air mixture exceeds $300 \mathrm{~m} / \mathrm{s}$. The peak pressure of the shock wave is $\sim 0.1$ 


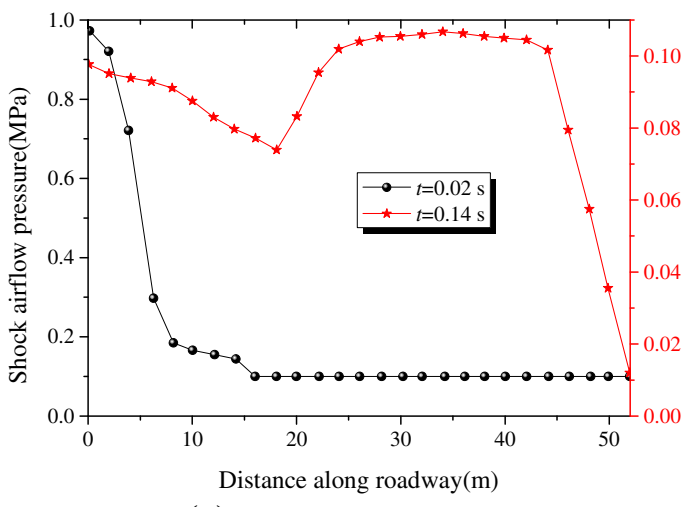

(a) Shock airflow pressure

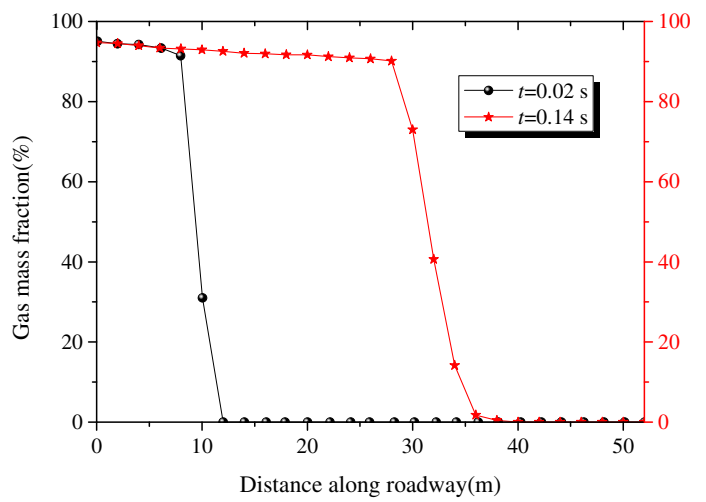

(b) Gas mass fraction

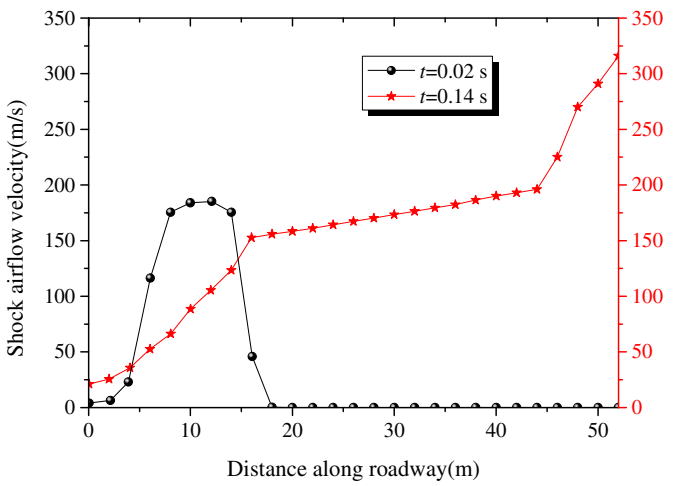

(c) Shock airflow velocity

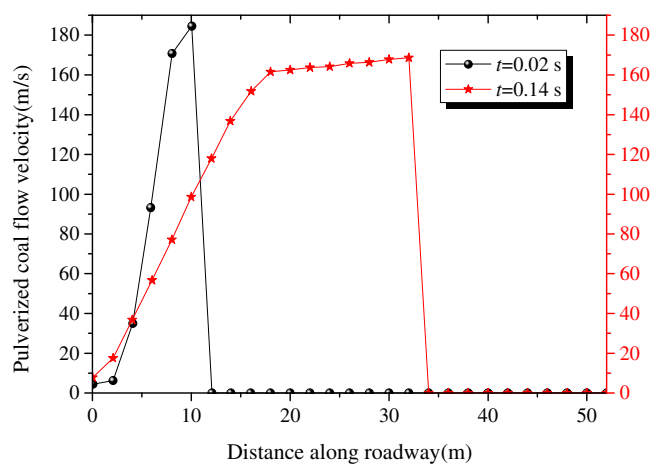

(d) Pulverized coal flow velocity

Fig. 3 Shock wave and coal-gas flow propagation in a straight roadway at different times following the outburst

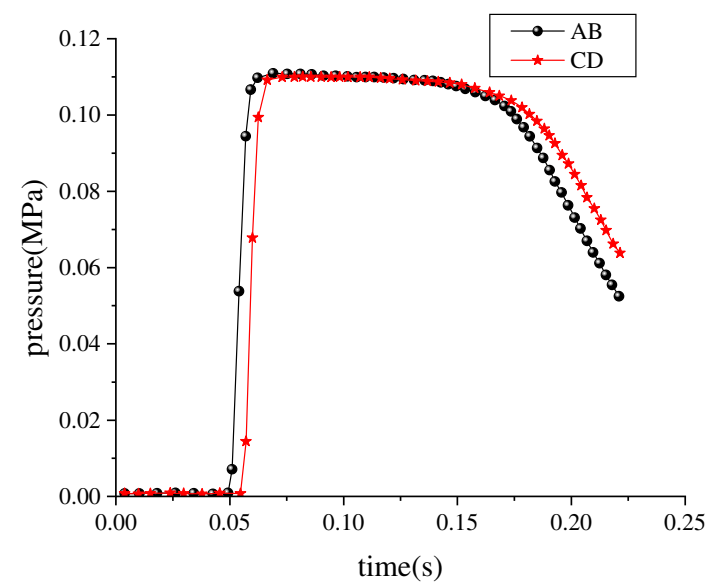

Fig. 4 Gas overpressure variation with time at two different cross sections

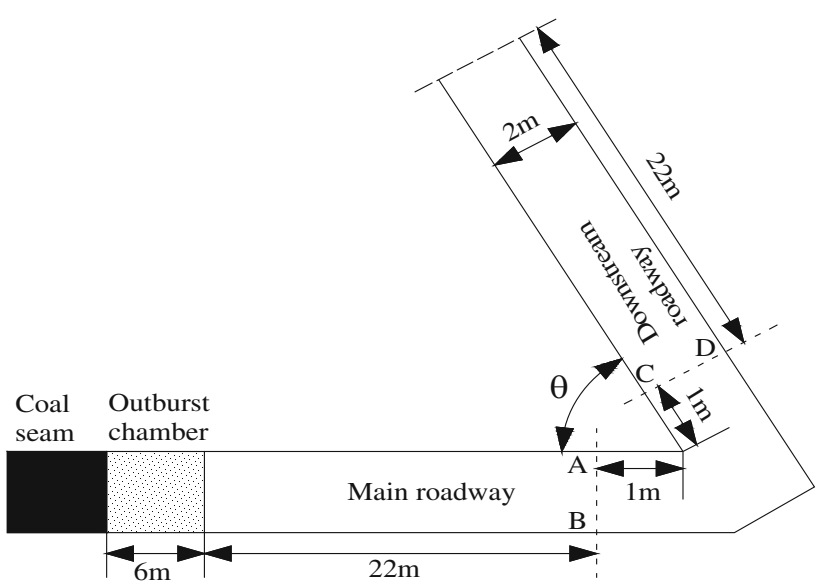

Fig. 5 Geometry of corner roadway

$\mathrm{MPa}$, and the average pressure attenuation speed is $0.02 \mathrm{MPa} / \mathrm{m}$. At this stage, the attenuation amplitude of the shock wave in the straight roadway is not greatly affected by the pulverized coal volume fraction of $5 \%$.

Apparent from the velocity distribution in the shock airflow and pulverized coal flow (Fig. 3c, d) is that slip velocity occurs between the mixture components. At $0.02 \mathrm{~s}$, the gas and pulverized coal flow is ejected uniformly from the outburst chamber. The velocity of the gas flow front reaches $\sim 190 \mathrm{~m} / \mathrm{s}$, with the velocity of the pulverized coal slightly lagging at $\sim 180 \mathrm{~m} / \mathrm{s}$. Thus, there is no significant difference in the velocities of the gas and the pulverized coal flows. By $0.14 \mathrm{~s}$, the gas has spread throughout the entire roadway. At this stage, the velocity of the shock front is $\sim 300 \mathrm{~m} / \mathrm{s}$, while the velocity of the pulverized coal remains $\sim 160 \mathrm{~m} / \mathrm{s}$ and is principally distributed in the first $3 / 5$ ths of the roadway. The velocity difference between the gas and the pulverized coal is now significant and results from the uneven pressure gradient and drag forces acting on the pulverized coal particles and 


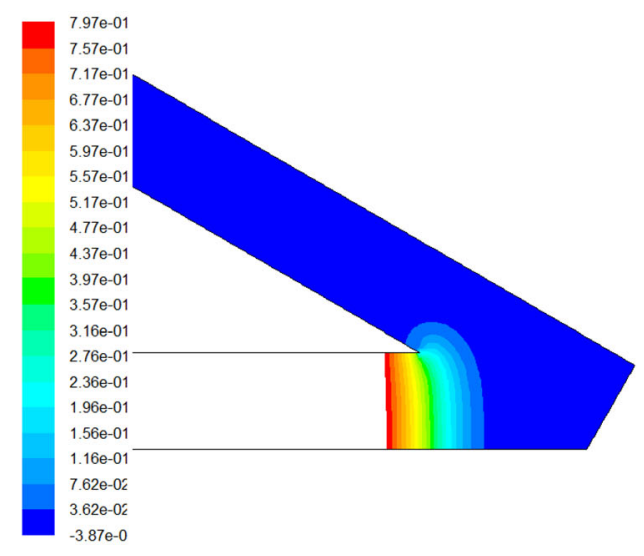

$t=0.050 \mathrm{~s}$

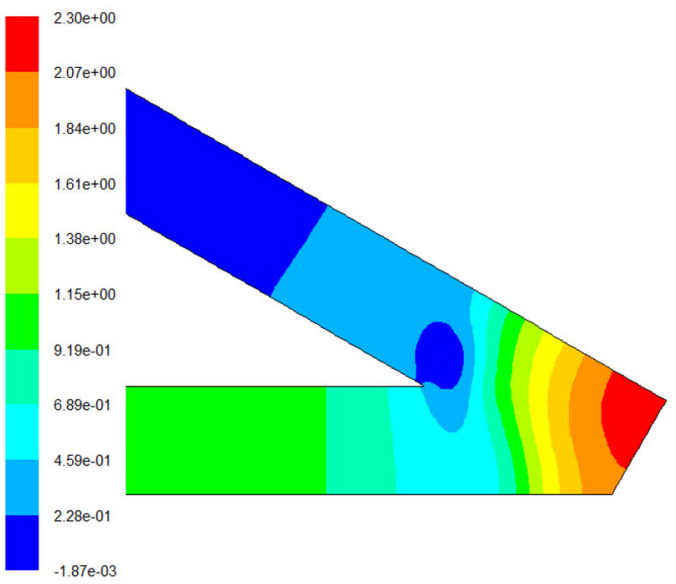

$t=0.065 \mathrm{~s}$

Fig. 6 Contour plots of outburst shock wave overpressure with time

resulting from collision between particles and the roadway wall.

The monitoring results from the two downstream sections are shown in Fig. 4. Pressures at sections $A B$ and $C D$ first increase rapidly at $\sim 0.05 \mathrm{~s}$ in the straight roadway and peak at $\sim 0.11 \mathrm{MPa}$ from 0.05 to $0.15 \mathrm{~s}$. Then the shock wave overpressure begins to decrease (initial pressure of the outburst chamber is $1 \mathrm{MPa}$ and the volume fraction of pulverized coal is 5\%). The attenuations of both pressure cycles are almost identical-indicating that a straight roadway with a uniform cross-section has little influence on the short-distance/time propagation of the outburst shock airflow. Furthermore, the overpressure reduction from section $A B$ to $C D$ is much smaller than the average attenuation velocity of the entire simulated roadway at $\sim 0.02 \mathrm{MPa} / \mathrm{m}$. Thus, the attenuation speed of shock wave is related to the distance from the outburst source.

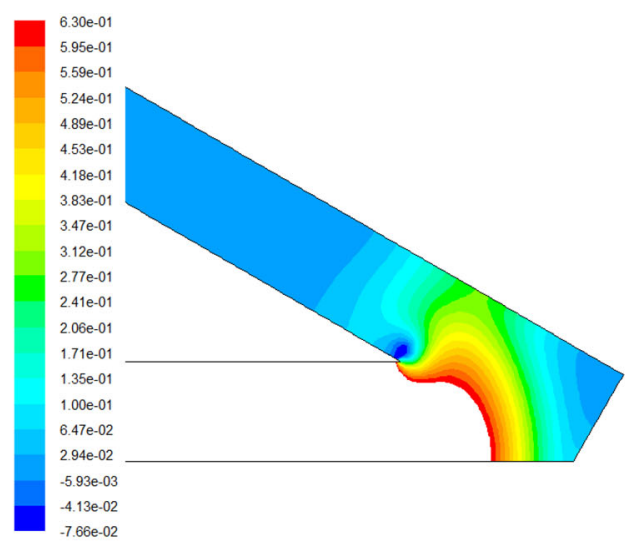

$t=0.055 \mathrm{~s}$

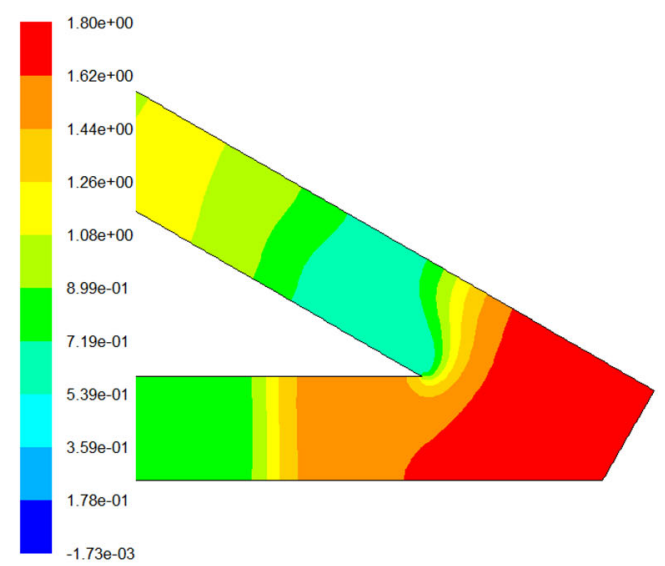

$t=0.090 \mathrm{~s}$

\subsection{Propagation characteristics in corner roadways}

The corner roadway geometry is shown in Fig. 5, and the angle of the corners is $\theta$. Two monitoring sections are at $22 \mathrm{~m}(A B)$ and $24 \mathrm{~m}(C D)$ downstream from the outburst chamber.

The results show that when $\theta$ is $30^{\circ}$, the front of the shock wave and the gas flow reaches the corner at $~$ $0.05 \mathrm{~s}$ (Fig. 6). The wave front is relatively flat and the pressure distribution distinct. By $0.055 \mathrm{~s}$, the front of the shock airflow bypasses the corner with the wave front distributed irregularly with a curved surface. The airflow movement is principally manifest as a flow passing around the near corner with part of the impact airflow reflected from the outer wall away from the corner. At $0.06 \mathrm{~s}$ a low pressure area appears at the corner of the inner wall and is superposed by the reflected shock wave from the outer wall of the corner. The frontal shock flow is sharply compressed and a local high pressure appears at the corner of the outer wall. At $0.09 \mathrm{~s}$ this phenomenon becomes more obvious 


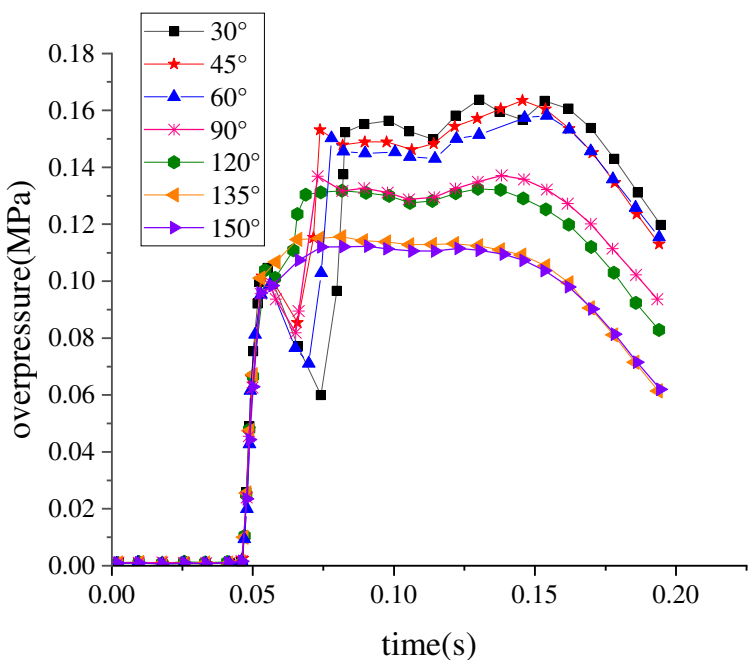

(a) Overpressure variation with time at section $A B$

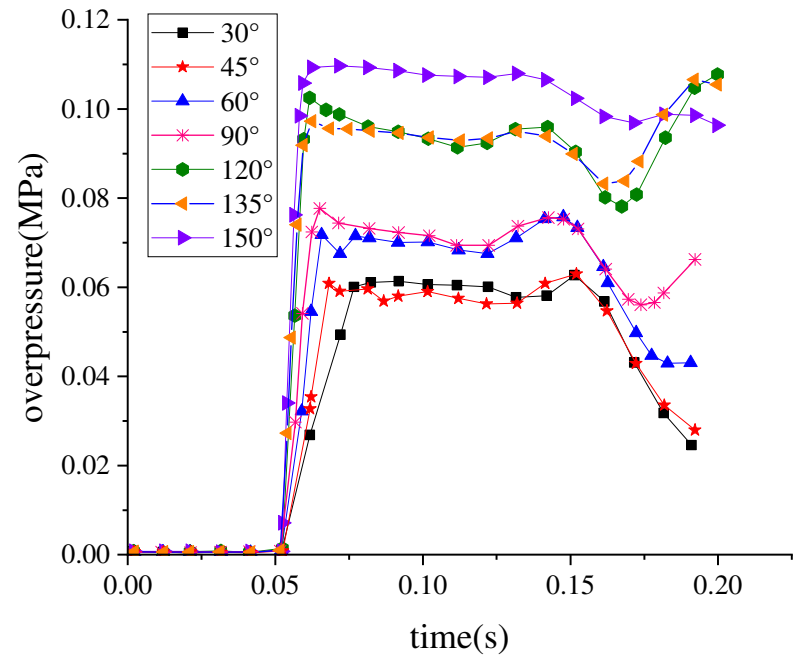

(b) Overpressure variation with time at section $C D$

Fig. 7 Gas overpressure variation with time at different cross sections and for different bend angles

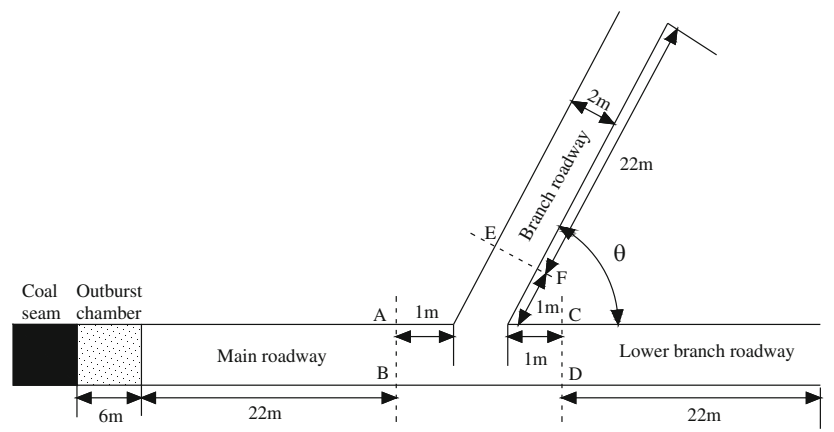

Fig. 8 Geometry of bifurcating roadway

and the outer corner presents a local high pressure and the pressure is stratified around the inner wall of the corner.

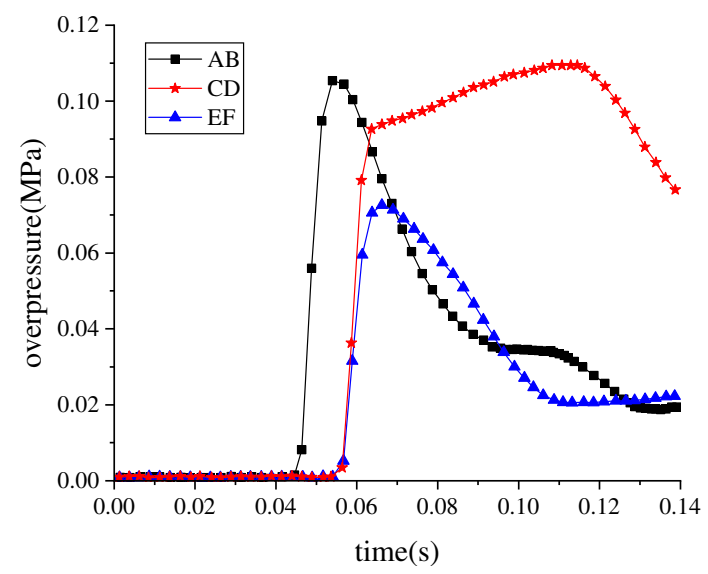

(a) $\theta=45^{\circ}$

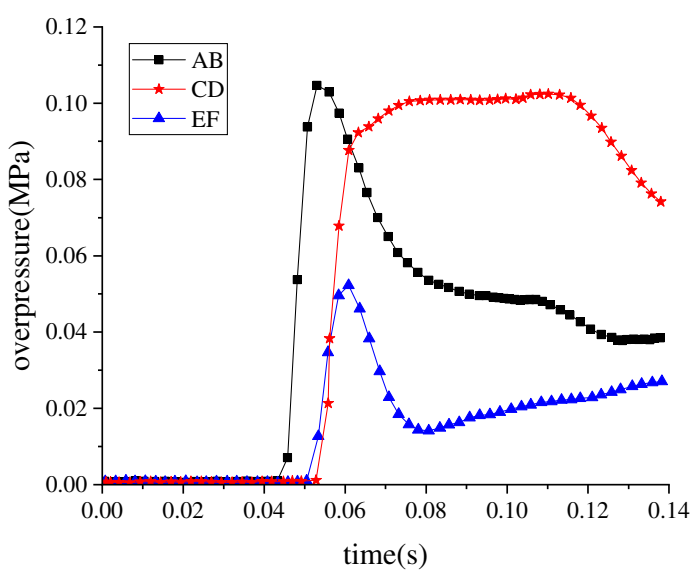

(b) $\theta=90^{\circ}$

Fig. 9 Gas overpressure variation with time for different bifurcation angles

When $\theta$ increases to $90^{\circ}$, comparing the shock airflow velocity distributions at three different times of $0.05,0.06$ and $0.16 \mathrm{~s}$, the high-speed airflow is principally along the inner wall of the corner and almost stagnates at the outer wall-as the shock waves and gas flows pass the corner. When the gas flows pass through the corner, a high-speed flow appears on the outer wall, and the pressure distribution is no longer obviously stratified at the corner. The wave front recovers to be flat after a certain distance through the corner. Thus, it takes some time for the wave front to recover.

The pressure variation at sections $A B$ and $C D$, representing roadways with different angles, is shown in Fig. 7. A peak in the secondary pressure at section $A B$ is barely apparent when the corner is more than $90^{\circ}$ and further diminishes as the angle increases to greater than $135^{\circ}$. The pressure variation for the $150^{\circ}$ corner roadway is similar to that of a straight roadway. Moreover, the smaller the angle, the larger the rebound in the second peak pressure, and the smaller the pressure trough between the two peak pressures. All these indicate that the sharper the corner, the 


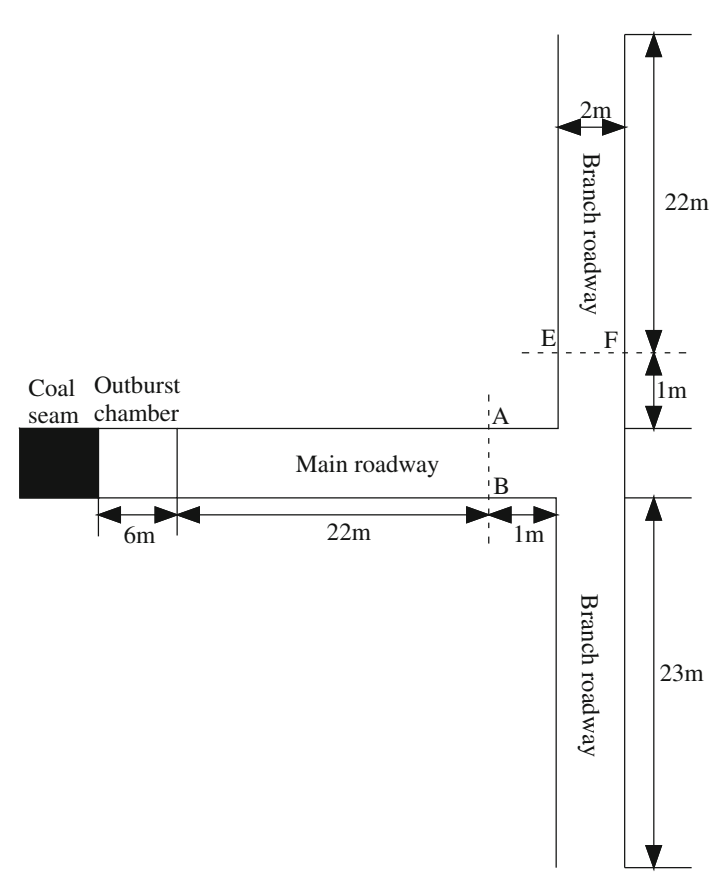

Fig. 10 Geometry of T-shaped roadway

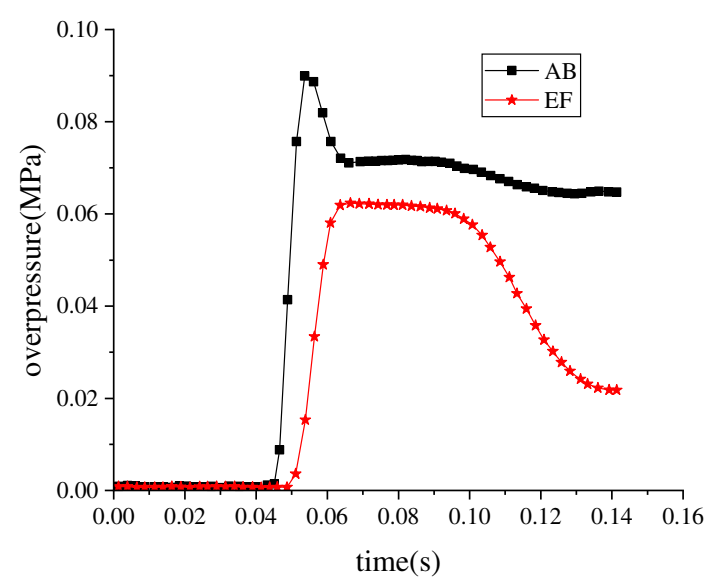

(a) T-shaped roadway

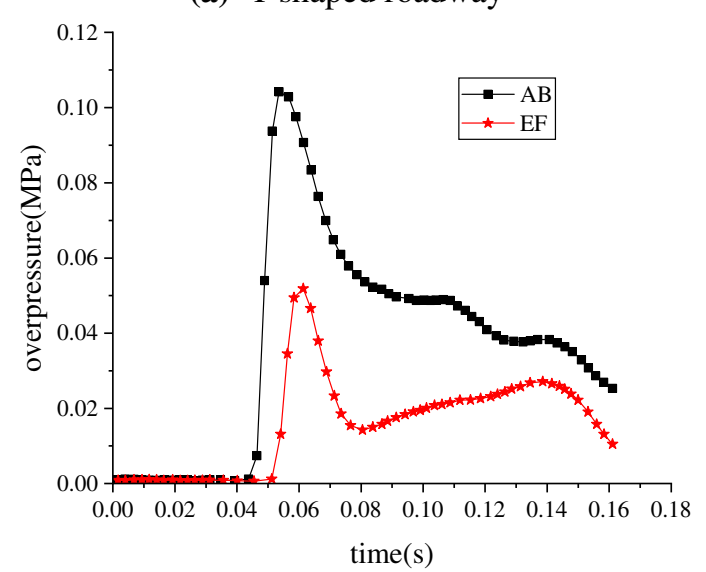

(b) $90^{\circ}$ symmetrical bifurcation roadway

Fig. 11 Comparison of variation in gas overpressure with time for similar roadway types more obvious the structural effect, and the longer the interval between the time of arrival of the two peak pressures. Similarly, the sharper the corner, the larger the peak pressure at section $C D$. For example, in the case of a $30^{\circ}$ corner, the pressure peak does not appear until $0.075 \mathrm{~s}$, while for a $90^{\circ}$ corner, the arrival time of the pressure peak is $0.060 \mathrm{~s}$, and the peak pressure arrival time is advanced by $0.055 \mathrm{~s}$ for a $150^{\circ}$ corner. Furthermore, the peak pressure at section $C D$ becomes smaller and the duration of overpressure becomes shorter with the decrease of corner angle.

\subsection{Propagation characteristics in bifurcating roadways}

The geometry of a bifurcating roadway is shown in Fig. 8 . In this, $\theta$ represents the bifurcation angle. The simulated conditions are consistent with those selected for the straight roadway, and monitoring sections are at $A B, C D$ and $E F$.

The overpressure at each monitoring section for different bifurcation angles shows that the overpressures of the shock waves at cross sections $A B$ and $E F$ are similar (Fig. 9), although the duration of the overpressure at $C D$ is $\sim 0.06 \mathrm{~s}$, much longer than that at $A B$ and $C D$. This is principally due to the delayed release of pressure caused by reflection at the bifurcation. The delay in release time is almost independent of the bifurcation angle. Shock wave overpressures in the lower branch roadway at section $C D$ are higher than those of the upper branch roadway at $E F$ but the arrival times are the same. The smaller the bifurcation angle, the more similar the change in overpressures at sections $E F$ and $A B$. The overpressure values at sections $C D$ and $E F$ are similar. When the roadway bifurcates at $90^{\circ}$, the overpressure at section $E F$ is much smaller than that at $C D$, from which it can be inferred that the shock wave and gas flows principally enter the lower branch roadway. Compared with the overpressure losses for the two bifurcation angles, the overpressure at section $C D$ is almost unchanged, and the peak value is $\sim 0.09 \mathrm{MPa}$, while it is reduced from $0.07 \mathrm{MPa}$ to $0.05 \mathrm{MPa}$ at section $E F$. It can be concluded that the larger the bifurcation angle, the more obvious the influence of roadway structure, and the smaller the overpressure in the bifurcated roadway.

\subsection{Propagation characteristics in T-shaped roadways}

The typical geometry of a T-shaped roadway is shown in Fig. 10. The simulated outburst conditions are the same as above. The characteristics of shock wave propagation are observed at sections $A B$ and $E F$.

The variations in the shock wave and gas flow overpressure at sections $A B$ and $E F$ are quite different for the 


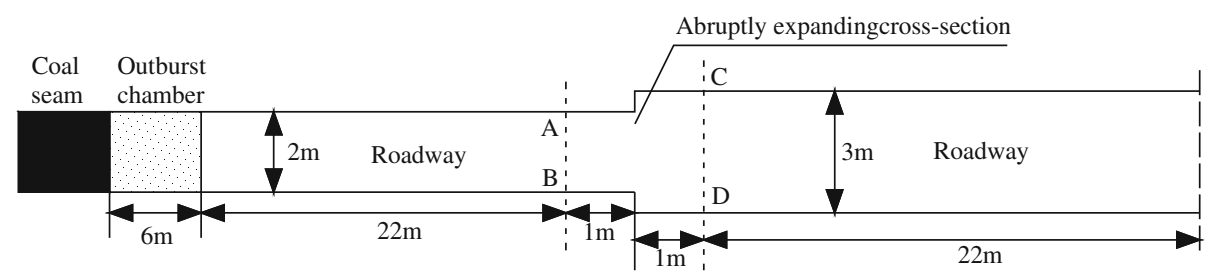

Fig. 12 Geometry of abruptly expanding roadway

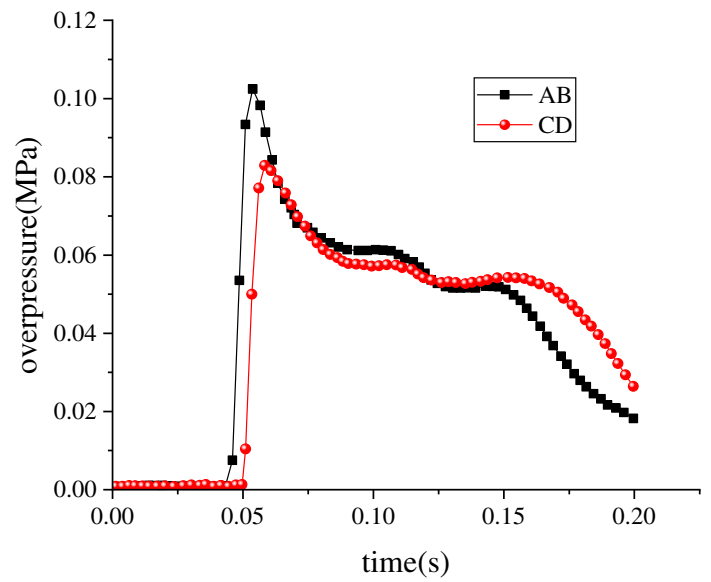

Fig. 13 Gas overpressure variation with time at different cross sections

two cases -namely, a T-shaped roadway and a $90^{\circ}$ symmetrical bifurcation roadway (Fig. 11). The pressure attenuation is slow, and the amplitude is small at section $A B$ in the T-shaped roadway, the same at section $E F$. Nevertheless, the attenuation of overpressure is completed only within $0.02 \mathrm{~s}$ for the $90^{\circ}$ symmetrical bifurcation case. The principal reason is that the reflecting and bypassing effect at the structural change of the T-shaped roadway is more dominant. High pressure can be maintained for a long time in the upstream and downstream branch roadways, while the $90^{\circ}$ symmetrical branch roadway has a bifurcated outlet in the same direction as the original airflow leading to the phenomenon that the overpressure peaks in the two bifurcated roadways are low, and the durations are short. In summary, the reflecting, bypassing phenomena and the blocking effect are more dominant in the T-shaped roadway, and the attenuation is more significant.

\subsection{Propagation characteristics in variable cross- section roadways}

An abruptly expanding roadway geometry is shown in Fig. 12, having a variable cross-section in a straight roadway. The height of the abruptly expanding roadway has been changed from 2 to $3 \mathrm{~m}$. The initial and boundary conditions for simulations are the same as above. Monitoring sections are at $A B$ and $C D$ to observe the characteristics of the propagation of the shock wave.

The results show that the shock wave and gas flows are principally distributed in the center of the conduit, dispersing to the two walls after they enter the abruptly expanding section-apparent from the velocity clouds of the outburst shock wave and gas flows. Furthermore, the bypassing phenomenon results in turbulent flow and a decrease in the propagation velocity at the variable crosssection. Apparent from Fig. 13 is that the attenuation of overpressure at monitoring section $A B$ is the same as that at $C D$. The attenuation rate of the overpressure peak is $0.02 \mathrm{MPa} / \mathrm{m}$, and the average attenuation speed is $0.01 \mathrm{MPa} / \mathrm{m}$ - less than that in straight roadway. In addition, the attenuation of overpressure is principally caused by the decrease of intensity per unit area in the shock wave when the cross-section becomes larger. Therefore, it can be considered that the abruptly expanding cross-section has little effect on the propagation of outburst shock waves.

\subsection{Propagation characteristics in blocked roadways}

The geometry of an outburst roadway blocked with an obstacle is shown in Fig. 14. The obstacle is $0.2 \mathrm{~m}$ wide and $h$ high. The height of straight roadway $H$ is $2 \mathrm{~m}$, and the obstacle "constriction rate" $R$ is defined as the ratio of $h$ to $H$. The initial and boundary conditions for simulation

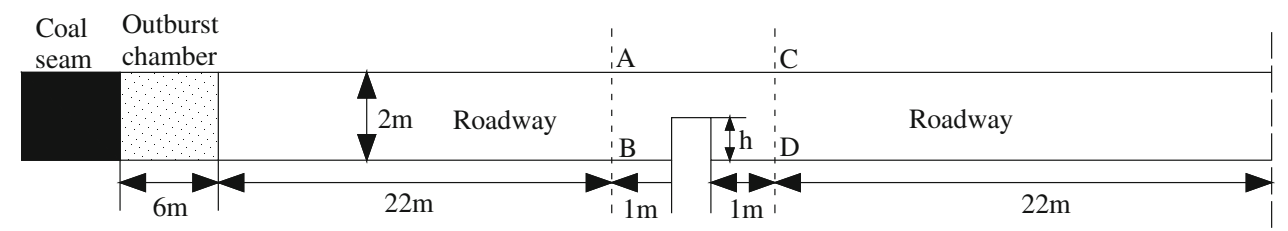

Fig. 14 Geometry of blocked roadway 


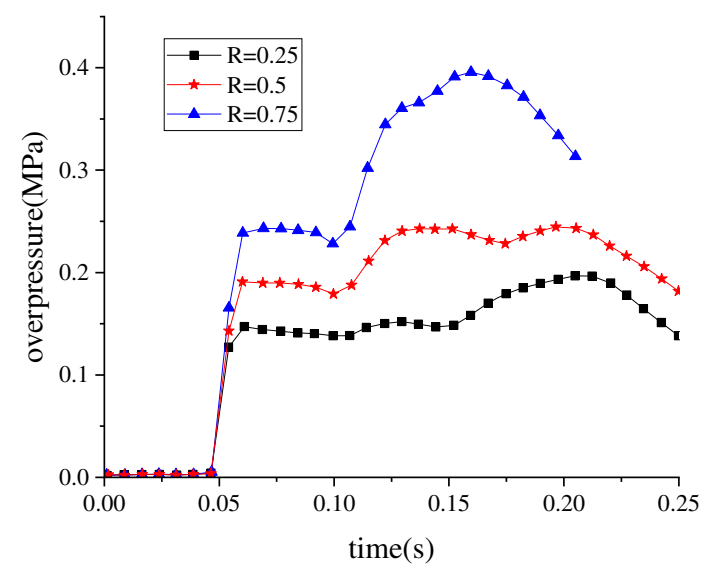

Section $\mathrm{AB}$

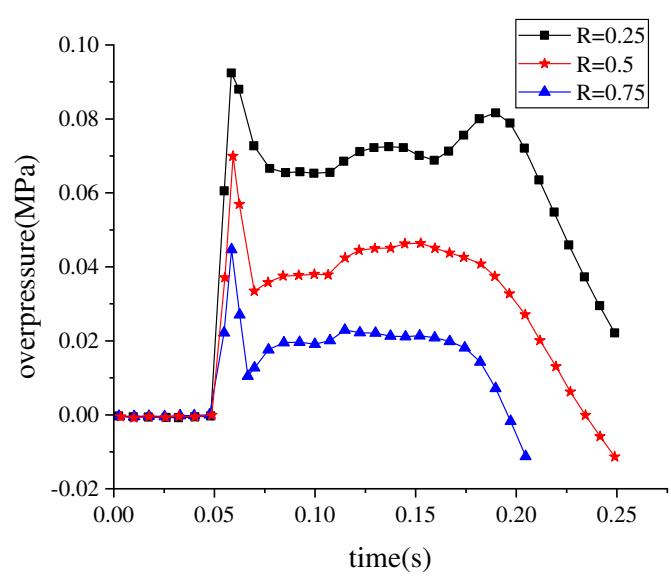

Section CD

Fig. 15 Gas overpressure variation with time at different sections and for different blocking ratios

Table 1 Attenuation coefficients of shock waves for different roadway types

\begin{tabular}{lllllll}
\hline Type of roadway & & $\begin{array}{l}\mathrm{AB} \\
(\mathrm{MPa})\end{array}$ & $\begin{array}{l}\mathrm{CD} \\
(\mathrm{MPa})\end{array}$ & $\begin{array}{l}\mathrm{EF} \\
(\mathrm{MPa})\end{array}$ & $\eta_{1}$ & $\eta_{2}$ \\
\hline Straight roadway & & 0.121 & 0.110 & - & 1.10 & - \\
Corner roadway & $30^{\circ}$ & 0.156 & 0.064 & - & 2.43 & - \\
& $45^{\circ}$ & 0.159 & 0.069 & - & 2.30 & - \\
& $60^{\circ}$ & 0.157 & 0.072 & - & 2.18 & - \\
& $90^{\circ}$ & 0.145 & 0.085 & - & 1.71 & - \\
& $120^{\circ}$ & 0.132 & 0.103 & - & 1.28 & - \\
& $135^{\circ}$ & 0.118 & 0.106 & - & 1.11 & - \\
& $150^{\circ}$ & 0.116 & 0.110 & - & 1.05 & - \\
Bifurcating roadway & $45^{\circ}$ & 0.106 & 0.091 & 0.072 & 1.16 & 1.47 \\
& $90^{\circ}$ & 0.104 & 0.094 & 0.052 & 1.11 & 2 \\
T-shaped roadway & & 0.091 & - & 0.072 & - & 1.26 \\
Abruptly expanding & & 0.103 & 0.084 & - & 1.23 & - \\
$\quad$ roadway & & & & & & \\
Blocked roadway & 0.25 & 0.146 & 0.093 & - & 1.57 & - \\
$\quad$ constriction rate $R$ ) & 0.5 & 0.190 & 0.046 & - & 4.13 & - \\
& 0.75 & 0.240 & 0.045 & - & 5.33 & - \\
\hline & & & & & &
\end{tabular}

are the same as above. Monitoring sections are at $A B$ and $C D$.

The simulations show that when the shock wave encounters the obstacle, it is obstructed directly in front of the obstacle, causing reflection, a sharp decrease in the velocity, and interference with the airflow coming towards the obstacle. A small part of the airflow passes around the obstacle along its tip, moving with high-speed. This part of the high-speed airflow has reached the upper right tip of the obstacle at $0.07 \mathrm{~s}$. Otherwise, the velocity of the airflow behind the obstacle is non zero, and the high-speed airflow tends to move along the upper right tip of the obstacle. This indicates that the bypassing effect and the diffraction of airflow each play an important role at the obstacle. Apparent from the overpressure curves at the monitoring sections in Fig. 15, the blocking effect is more obvious for the shock wave and both gas flows and the reflection interference are stronger when the obstacle constriction rate is larger. At section $A B$, the local pressure peak increases sharply when the constriction rate reaches to 0.75 . Therefore, the greater the obstacle constriction rate at section $C D$, the greater the pressure peak attenuation.

\section{Comparison of outburst attenuation coefficients for different roadway types}

As shown in Table 1 , shock wave overpressures $P_{\mathrm{AB}}, P_{\mathrm{CD}}$ and $P_{\mathrm{EF}}$ are measured at sections $A B, C D, E F$ respectively. $\eta_{1}$ is the ratio of $P_{\mathrm{AB}}$ to $P_{\mathrm{CD}} \cdot \eta_{2}$ is the ratio of $P_{\mathrm{AB}}$ to $P_{\mathrm{EF}}$. The attenuation coefficients of roadways with corners, bifurcations and obstacles are larger than straight roadways. The influence of roadway structure becomes negligible after the corner angle increases to $135^{\circ}$. Compared to straight roadways, the coefficient does not change significantly when the obstacle constriction rate $R$ is 0.25 , but increases sharply with an increase in $R$. The T-shaped and the abruptly expanding roadways exert little influence on shock waves. These are important parameters for simplifying transport in complex roadway networks in the farfield.

\section{Experimental studies of shock wave attenuation at outburst sources}

We developed an experimental system (Fig. 16) consisting of an outburst chamber, simulated roadways, pressure sensors and a data acquisition system. The outburst chamber is a cylindrical device with a net diameter of $300 \mathrm{~mm}$ and a height of $450 \mathrm{~mm}$. This is equipped with a three-way valve, a vacuum gauge and a shock-proof pressure gauge. It is linked to the simulated roadways, serving 


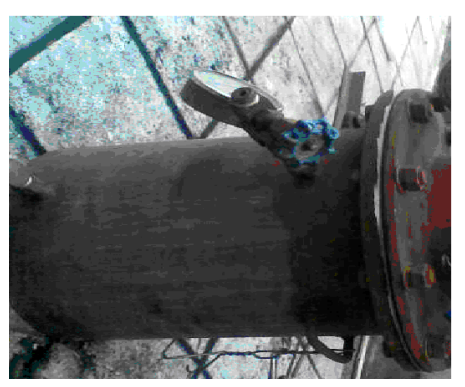

(a) Outburst chamber

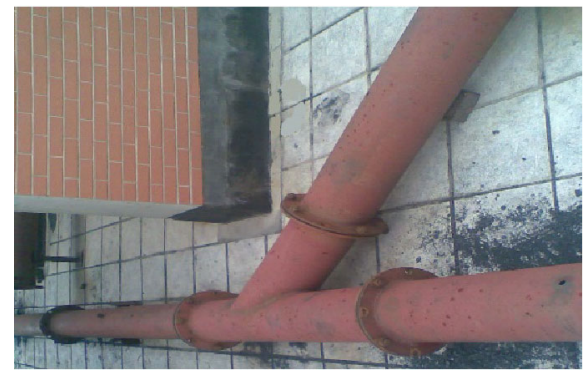

(b) Experimental roadways

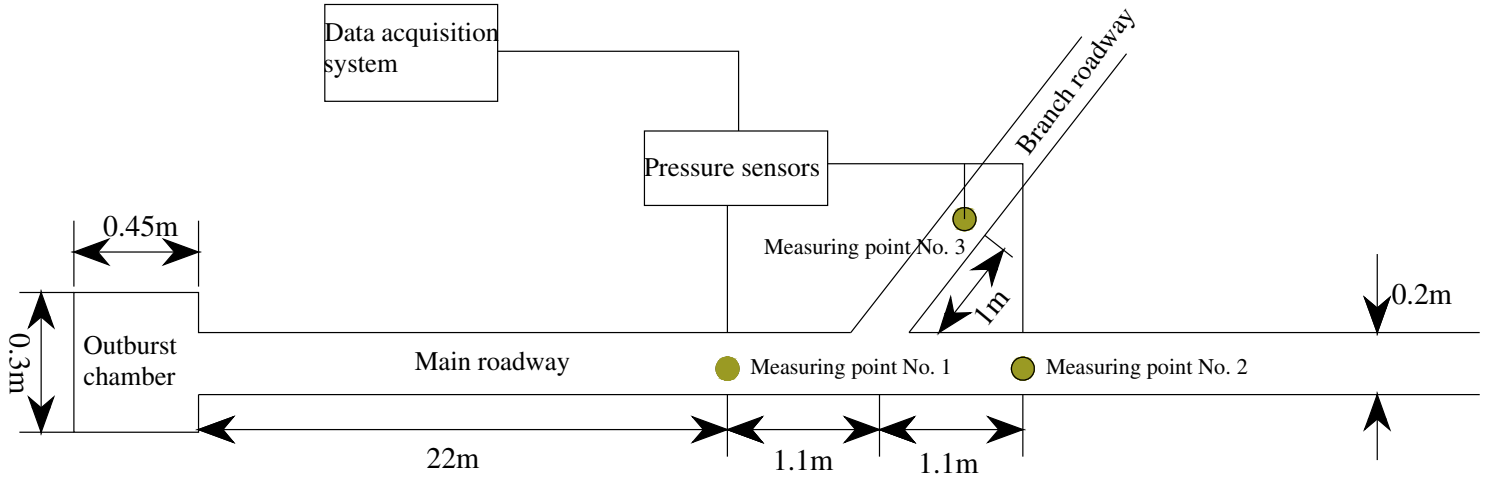

(c) Layout of pressure sensors in experimental roadways

Fig. 16 Experimental devices for simulating shock waves of outburst

as an energy source for outbursts. The simulated roadways comprise circular pipes of diameters $200 \mathrm{~mm}$ and $300 \mathrm{~mm}$ (for simulating roadways with abruptly expanding sections) and lengths of $0.5,1.0,1.5$ and $2.0 \mathrm{~m}$ connected by sealing flanges. CYG series pressure transducers with both touch sensors and signal processing are selected as pressure sensors to collect the dynamic pressure signal of shock waves arriving at monitoring points. The data acquisition system adopts a TDEC dynamic signal recorder and analyzer to process, display and store the transmission signal. In the experiment, pressurized film is used to induce the outburst, which can simulate the different initial pressures of outburst shock waves. The pressure sensors are installed in the roadways, at locations $A B, C D$ and $E F$ in the numerical simulations. They are identified as measuring points, No. 1, 2 and 3, respectively.
Table 2 shows the attenuation coefficients for shock waves in the case of a straight roadway, a $45^{\circ}$ bifurcated roadway, a $90^{\circ}$ bifurcated roadway and an abruptly expanding roadway. $p_{1}, p_{2}$ and $p_{3}$ are the peak pressures of the outburst shock waves at measuring points 1,2 and 3 respectively. $k_{12}$, is the attenuation coefficient between points 1 and 2 calculated from the ratio $p_{1}$ to $p_{2} . k_{13}$, the attenuation coefficient between points 1 and 3 calculated from the ratio $p_{1}$ to $p_{3}$. Comparing Tables 1 and 2, the peak pressures of the outburst shock wave calculated by the numerical models are higher than those recovered from the experiments. This is likely because gas desorption and the pulverization of coal are not considered in the numerical simulations-rather, it is assumed that the pulverized coal and gas have been evenly mixed and are ready to be released before the outburst occurs. Indeed, a portion of the 
Table 2 Experimentally determined outburst attenuation coefficients for different roadway types

\begin{tabular}{llllll}
\hline Type of roadway & Pressure for outburst $(\mathrm{MPa})$ & $p_{1}(\mathrm{MPa})$ & $p_{2}(\mathrm{MPa})$ & $p_{3}(\mathrm{MPa})$ & $k_{12}$ \\
\hline Straight roadway & 1.00 & 0.050 & 0.042 & - & 1.20 \\
$45^{\circ}$ bifurcation roadway & 1.00 & 0.046 & 0.038 & 0.030 & 1.21 \\
$90^{\circ}$ bifurcation roadway & 1.00 & 0.062 & 0.054 & 0.028 & 1.53 \\
Abruptly expanding roadway & 1.00 & 0.053 & 0.041 & - & 2.21 \\
\hline
\end{tabular}

energy is consumed to transport the pulverized coal, and it takes a finite time for absorbed gas to desorb into free gas in the experiment. However, the overpressures and their timing, and especially the trends, are similar between simulations and experiments. The calculated attenuation coefficients of various types of roadways are almost as high as in the simulations, verifying the reliability of the numerical simulations in describing the attenuation laws for shock waves.

\section{Conclusions}

(1) The near-source attenuation characteristics of outburst shock waves for different roadway geometries and intersections are explored using a two-phase gas-solid flow model. Shock wave attenuation is weak in straight and abruptly expanding roadways, but more significant in T-shaped roadways, more significant in roadways with corners and bifurcations, and greatest in obstructed roadways.

(2) Different from a straight roadway, the shock wave overpressure does not follow a simple linear or exponential attenuation when the roadway structure is complex. Rather, these complex roadways produce local compression including secondary overpressure peaks in roadways with obstacles and small corners (angles less than $135^{\circ}$ ).

(3) Compared with straight roadways, the attenuation coefficients for shock waves are larger in roadways with corners, bifurcations and obstacles, but the effect of structure becomes negligible when the corner angle increases to $135^{\circ}$. Shock waves are little attenuated in T-shaped and abruptly expanding roadways.

Acknowledgements This research is financially supported by the State Key Research Development Program of China (Grant No. 2018YFC0808101), the National Natural Science Foundation of China (51774292, 51874314, 51604278, 51804312), the Yue Qi Distinguished Scholar Project, China University of Mining \& Technology, Beijing, the Yue Qi Young Scholar Project, China University of Mining \&Technology, Beijing.

\section{Compliance with ethical standards}

Conflict of interest The authors declare no conflict of interest.

Open Access This article is licensed under a Creative Commons Attribution 4.0 International License, which permits use, sharing, adaptation, distribution and reproduction in any medium or format, as long as you give appropriate credit to the original author(s) and the source, provide a link to the Creative Commons licence, and indicate if changes were made. The images or other third party material in this article are included in the article's Creative Commons licence, unless indicated otherwise in a credit line to the material. If material is not included in the article's Creative Commons licence and your intended use is not permitted by statutory regulation or exceeds the permitted use, you will need to obtain permission directly from the copyright holder. To view a copy of this licence, visit http://creativecommons. org/licenses/by/4.0/.

\section{References}

ANSYS (2017). FLUENT theory guide. Release 17. ANSYS Inc, Canonsburg

Berger S, Sadot O, Ben-Dor G (2009) Experimental investigation on the shock-wave load attenuation by geometrical means. Shock Waves 20(1):29-40

Biamino L, Igra O, Jourdan G, Houas L (2013) Effect of an impinging shock wave on a partially opened door. Shock Waves 24(2):115-121

Chaudhuri A, Hadjadj A, Sadot O, Ben-Dor G (2012) Numerical study of shock-wave mitigation through matrices of solid obstacles. Shock Waves 23(1):91-101

Cheng W, Chen G (2000) Formation of coal and gas outburst shock wave and model establishment. Safety Coal Mines 9:23-25

Cheng W, Liu X, Wang K, Li X (2004) Study on regulation about shock-wave-front propagating for coal and gas outbursts. J China Coal Soc 1:57-60

Guo H, Cheng Y, Ren T, Wang L, Yuan L, Jiang H, Liu H (2016) Pulverization characteristics of coal from a strong outburst-prone coal seam and their impact on gas desorption and diffusion properties. J Nat Gas Sci Eng 33:867-878

Igra D, Igra O (2016) Shock wave mitigation by different combination of plate barriers; a numerical investigation. Eur J Mech B/Fluids 59:115-123

Jin K, Cheng Y, Ren T, Zhao W, Tu Q, Dong J, Wang Z, Hu B (2018) Experimental investigation on the formation and transport mechanism of outburst coal-gas flow: Implications for the role of gas desorption in the development stage of outburst. Int J Coal Geol 194:45-58

Lin B-q, Guo C, Sun Y-m, Zhu C-j, Hong Y-d, Yao H (2016) Effect of bifurcation on premixed methane-air explosion overpressure in pipes. J Loss Prevent Proc 43:464-470 
Miao F, Sun D, Hu Q (2013) The formation mechanism of shock waves in the coal and gas outburst process. J China Coal Soc 3:367-372

Otuonye F, Sheng J (1994) A numerical simulation of gas flow during coal/gas outburst. Geotech Geol Eng 1:15-34

Sun H, Cao J, Li M, Zhao X, Dai L, Sun D, Wang B, Zhai B (2018) Experimental Research on the Impactive Dynamic Effect of GasPulverized Coal of Coal and Gas Outburst. Energies 11(4):797

Uystepruyst D, Monnoyer F (2015) A numerical study of the evolution of the blast wave shape in rectangular tunnels. J Loss Prevent Proc 34:225-231

Wang K, Yu Q (2005) Nonlinear characteristics and prediction model of coal and gas outbursts. Xuzhou, China

Wang K, Zhou A, Zhang J, Zhang P (2012) Real-time numerical simulations and experimental research for the propagation characteristics of shock waves and gas flow during coal and gas outburst. Safety Sci 50(4):835-841

Wang K, Zhou A, Zhang J, Zhang P, Li C (2011) Study of the shock wave propagation and gas flow during a coal and gas outburst at the roadway with a right-angled bend. J China U Min Technol 6:858-862

Wu X, Peng Y, Xu J, Yan Q, Nie W, Zhang T (2019) Experimental study on evolution law for particle breakage during coal and gas outburst. Int J Coal Sci Technol 7(1):97-106
Xu L, Jiang C (2017) Initial desorption characterization of methane and carbon dioxide in coal and its influence on coal and gas outburst risk. Fuel 203:700-706

Xue S, Wang YC, Xie J, Wang G (2011) A coupled approach to simulate initiation of outbursts of coal and gas - Model development. Int J Coal Geol 86(2-3):222-230

Xue S, Yuan L, Wang J, Wang Y, Xie J (2015) A coupled DEM and LBM model for simulation of outbursts of coal and gas. Int J Coal Sci Technol 2(1):22-29

Yang S, Zhang R (2012) Research on injuries due to shock wave and gas flow from coal and gas outburst. China Safety Sci J 11:62-66

Yu Q (1992) Coal mine gas control. Xuzhou, China

Zhao B, Wen G, Sun H, Sun D, Yang H, Cao J, Dai L, Wang B (2018) Similarity criteria and coal-like material in coal and gas outburst physical simulation. Int J Coal Sci Technol 5(2):167-178

Zhao W, Wang K, Zhang R, Dong H, Lou Z, An F (2020) Influence of combination forms of intact sub-layer and tectonically deformed sub-layer of coal on the gas drainage performance of boreholes: a numerical study. Int J Coal Sci Technol. https://doi.org/10. 1007/s40789-020-00317-0

Zhou A, Wang K (2017) A transient model for airflow stabilization induced by gas accumulations in a mine ventilation network. J Loss Prevent Proc 47:104-109 